\title{
Friction and Visco-Elasticity Effects in Tendon-based Transmission Systems
}

\author{
G. Palli, G. Borghesan and C. Melchiorri \\ DEIS - Dipartimento di Elettronica, Informatica e Sistemistica \\ Università di Bologna \\ Viale Risorgimento 2, 40136 Bologna, Italy \\ \{gianluca.palli, gianni.borghesan, claudio.melchiorri\}@unibo.it
}

\begin{abstract}
In this paper, the characterization of the force distribution along a tendon sliding on a curved pathway, subject to friction and visco-elastic effects, is investigated. In order to have a better understanding of the system behavior, a specific setup able to measure tension forces in different points along the tendon's path has been built. Experimental data collected by measuring the tendon tension forces during both the pulling and the release phase are presented, and theoretical models reproducing the tendon behavior with increasing fidelity are proposed. In particular, the friction arising from the interaction between the tendon pathway and the tendon itself is characterized by means of a LuGre-like dynamic friction model. The introduction of a dynamic friction model allows to reproduce in simulation some effects arising during experimental activities that cannot be reproduced employing an equivalent static friction model. Moreover, the adoption of tendons made by polymeric fibers introduces hysteresis in the tendon transmission characteristic due to the plasticity and creep phenomena typical of these materials. With the aim of reproducing this behavior, a visco-elastic model is used for modeling the tendon compliance.
\end{abstract}

Index Terms-Tendon Transmission, Friction Modeling, Compliant Actuation, Nonlinear Systems.

\section{INTRODUCTION}

Tendon-based transmission systems represent a profitable method to transmit actuation forces in robotic devices. This is particularly true if the motors, because of requirements on inertia and size of the device, are placed remotely with respect to the joints. The use of tendons in general allows also a simpler mechanical design with respect to other solutions based on leverages, transmission shafts or gears.

Tendon-based transmission systems have been successfully employed in several robotic devices, such as endeffectors for surgical robots [1], [2], haptic interfaces, [3], robotic hands, see e.g. [4], [5], [6], [7], [8], and are currently employed in the design of an innovative robotic hand whose main features are described in [9]. A prototype of the new tendon-driven robotic finger is shown in Fig. 1.

Although tendon-based transmission systems present various advantages, some intrinsic limitations are introduced mainly due to the unidirectionality of the actuation forces and to the limited stiffness of the tendons. Also the problem of routing of the tendons from the motors to the joints must be solved and two main approaches can be adopted:

This research has been partially funded by the EC Seventh Framework Programme (FP7) under grant agreement no. 216239 as part of the IP DEXMART (DEXterous and autonomous dual-arm/hand robotic manipulation with sMART sensory-motor skills: A bridge from natural to artificial cognition)

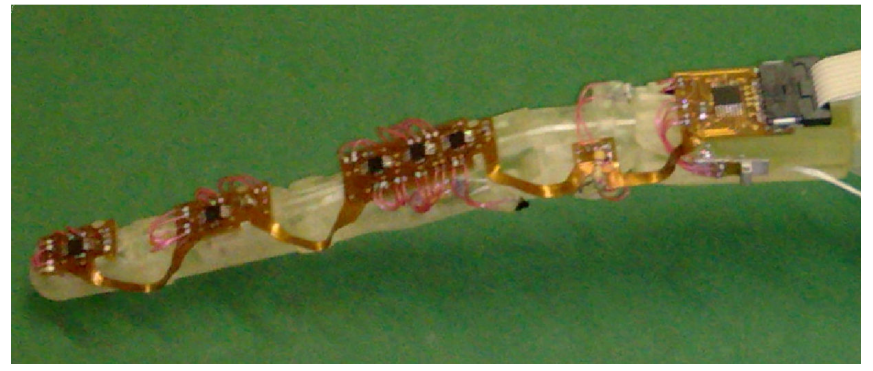

Fig. 1. A tendon-driven robotic finger prototype.

pulley-based routing and fixed path (e.g. sheaths, tubes or canals). While the use of pulleys strongly reduces the effect of friction but requires suitable preload mechanisms for preventing the tendon route-off and limits the benefits due to the simplified mechanical design and physical dimensions, the routing solution based on fixed path allows the maximum design simplification and flexibility but introduces distributed friction along the tendon, arising from the interaction between the tendon and a curved path. A detailed investigation of the friction phenomena in tendon-sheath transmission systems can be found in [10], [11], [12]. The adoption of fixed path for routing the tendons is justified, besides by mechanical design issues, by the possibility of compensating for friction side effects by means of suitable control strategies [13].

Another important issue is related to the choice of the tendons' material. Very thin steel ropes have often been used for the implementation of tendon-based transmission systems. This choice introduces a purely elastic behavior of the tendon but may present some difficulties in the device assembly due the need of suitable fixing and the limited curvature radius. More recently, the use of polymeric fibers [9] allows more flexibility in the implementation of tendon-based transmission systems due the large variety of materials and characteristics. On the other hand, these materials introduce plasticity and creeping in the force deformation characteristic of the tendon [14], as experimentally observed in [13].

With the aim of improving the knowledge of tendon-based transmission systems, the research activity reported in [13] has been further developed in this paper, from the point of view of both theoretical models and experiments. Several models with increasing accuracy in the system behavior rendering are proposed. Starting from the simplified model used for the design of the tendon friction compensation law 
presented in [13], more complete models of the tendon-based transmission are introduced, that describe also dynamical effects such as tendon plasticity. The friction effects, acting along the tendon, have been modeled by means of a modified version of the LuGre friction model. This friction model has been suitably developed to cope with the characteristics of the tendon transmission system. An experimental setup, equipped with seven force sensors able to measure the tendon tensions in different points along the tendon path, has been developed in order to observe the tendon force distribution during the working cycle. The experimental data are compared with simulation results, and the capabilities of the proposed models in rendering the predominant phenomena observed during experiments are highlighted.

\section{Modeling a Tendon Transmission System}

This section takes into account the modeling of the transmission characteristic of a tendon-based driving system with fixed routing path. In this system, the relation between the force applied to an extremity of the tendon (the input side) by the actuator (e.g. a rotative or linear electric motor) and the force applied on the other one (the output side) to the load (e.g. a joint of a robotic finger) is affected by the friction arising from the relative movement between the tendon and the path surface. Moreover, This transmission system exhibits also nonlinear elasticity, i.e. an hysteresis in the force/elongation characteristic, caused mainly by the friction and the plasticity of polymeric fibers.

Considering an infinitesimal tendon element, see Fig. 3, the constitutive equations that describe the system in quasistatic equilibrium condition are:

$$
\begin{aligned}
f_{n} & =T d \theta=T \frac{d x}{R} \\
d T & =-f_{f}
\end{aligned}
$$

where $d \theta$ is the angle subtended by the arc of length $d x, R$ is the radius of curvature, $\mu$ is the friction coefficient, $T$ and $d T$ are respectively the tendon tension and the infinitesimal tension decay, $f_{f}$ is the friction force and $f_{n}$ is the normal load due to the combined effects of the tendon tension and of the curvature imposed by the surface the tendon is sliding on. The contribution on the normal force $f_{n}$ due to gravitational effects are negligible because of the limited tendon mass.

If a tendon with purely elastic behavior is considered, the tendon deformation characteristic can be described as:

$$
\delta=\frac{1}{E A} T=\rho T
$$

where $E, A, \delta$ and $\rho$ are respectively the Young modulus, the cross sectional area, the elongation and the elasticity (normalized with respect to the length) of the tendon. It can be then noted that the tendon deformation is affected by the tendon tension decay due to the friction phenomena.

\section{A. Tendon Friction Model}

In [15] a Coulomb-based model of the friction phenomena acting on the tendon has been firstly introduced and in [13] this model has been used to design a feedforward friction

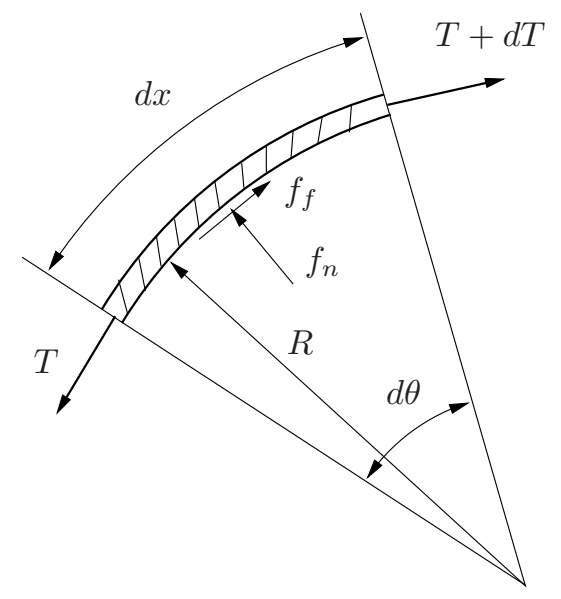

Fig. 3. Equilibrium of an infinitesimal tendon element.

compensation law for a tendon-based transmission system. This model can be written as:

$$
f_{f}=\mu f_{n} \operatorname{sign}(v)
$$

where $\mu$ is the friction coefficient and $v$ is the tendon element velocity.

In this work, the friction model has been improved by introducing a LuGre-like dynamic model [16]. In its original formulation [17], this model considers a constant normal force between two bodies in relative motion. This model has been then modified to consider a varying load force, and a configuration dependent friction coefficient has been introduced. The model considered here can be then represented as:

$$
\begin{aligned}
\dot{f}_{s} & =\sigma_{0}\left(v-\frac{f_{s}}{f_{n} \mu}|v|\right) \\
\mu & =\mu_{d}+\left(\mu_{c}-\mu_{d}\right) e^{-\frac{|v|}{\omega}} \\
f_{f} & =f_{s}+f_{v}=f_{s}+b v
\end{aligned}
$$

where $f_{s}$ and $f_{v}$ are respectively the static and viscous friction forces, $\mu_{d}$ and $\mu_{c}$ are the friction and stiction coefficients respectively and $\omega$ is the Stribeck velocity [16]. The friction coefficient $\mu_{d}$ can be easily identified by means of suitable experiments, as reported in [13], while the parameters $\mu_{c}, \rho_{0}$ and $\omega$ have been defined by comparing the simulative and experimental results, as reported in Sec. IV-B.

In [15], an analytic solution for the integration of (1)(4) along the tendon curvature in the two limit conditions in which the tendon velocity $v$ has the same sign (either positive or negative) along the whole tendon length has been given. The same result can be obtained in the same conditions by considering (5) instead of (4). The behavior of the tendon transmission system when the limit conditions mentioned above are not satisfied has been investigated in [13]. It is also important to mention that, as stated in [15], [13], the total force loss $F_{f}$ between the input and the output side of a tendon sliding on a curved surface depends only on the friction coefficient $\mu$ and on the total curvature angle $\theta$ of the path. 


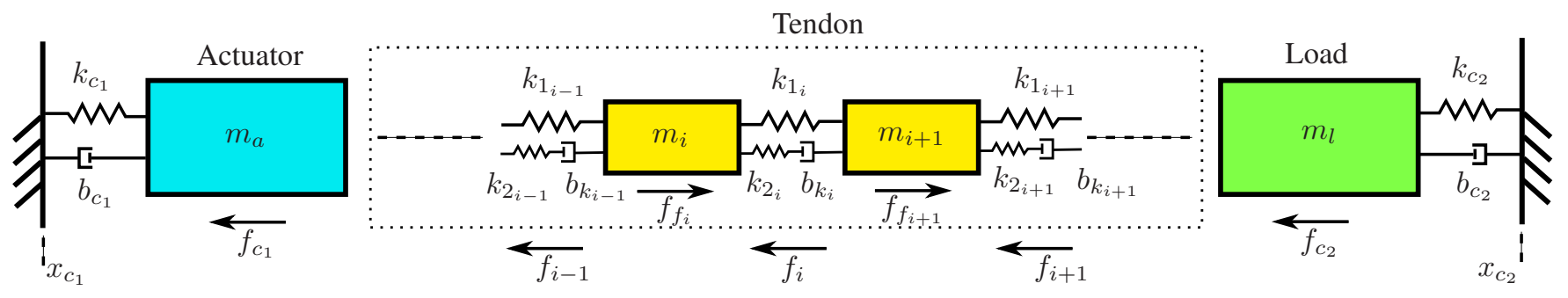

Fig. 2. Scheme the lumped-parameter tendon dynamic model.

\section{B. Visco-Elastic Tendon Model}

In previous works, the tendon has been modeled with a purely elastic force/elongation characteristic. In this case, the transfer function $G_{s}(s)$ between the tendon elongation velocity $V_{e}(s)$ and the elastic force $F_{e}(s)$ can be written as:

$$
G_{s}(s)=\frac{F_{e}(s)}{V_{e}(s)}=\frac{k}{s}
$$

where $k$ is the tendon stiffness. If the plasticity phenomena typical of polymeric materials is taken into account, the force/elongation characteristic of the tendon is modeled as in Fig. 2, and the correspondent transfer function is:

$$
G_{d}(s)=\frac{F_{e}(s)}{V_{e}(s)}=\frac{b_{k}\left(k_{1}+k_{2}\right) s+k_{1} k_{2}}{b_{k} s^{2}+k_{2} s}
$$

where $k_{1}$ is the tendon stiffness in static condition, $k_{1}+k_{2}$ is the stiffness at high frequency, and $b_{k}$ rules the transient time constant.

In Fig. 4 the experimental evaluation of the force/elongation characteristic of the tendon is reported: this plot shows the hysteresis of the deformation characteristic and the ripple in the motor position due to the combined effect of a limited displacement and the actuator friction.

\section{Lumped-Parameter Tendon Model}

The tendon transmission system has been simulated by means of a lumped-parameter tendon model, see the block scheme depicted in Fig. 2. In this model, the tendon has been considered as a chain of masses $m_{i}, i=1, \ldots, N$, being

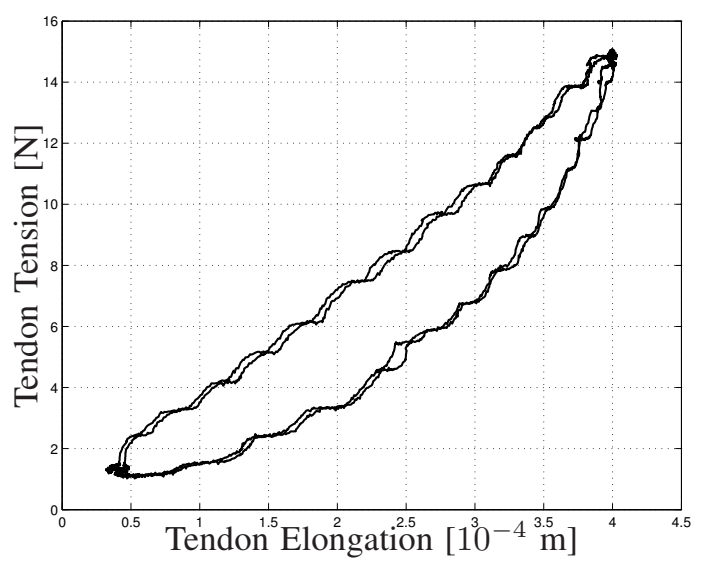

Fig. 4. Force/elongation characteristic of the tendon.
$N$ the number of tendon parts, connected by visco-elastic elements described by (7) (which interaction force is $f_{i}$ ) and subject to a friction force represented by (5) (represented by $f_{f_{i}}$ ). The actuator and the load are connected to the input and the output side of the tendon, modeled as the two masses $m_{a}$ and $m_{l}$ connected to a reference position $x_{c_{1}}$ and $x_{c_{2}}$ by means of a spring-damper system, and subject to imposed external forces, $f_{c_{1}}$ and $f_{c_{2}}$ respectively.

Past research activities show that a value $N \geq 40$ should be used to obtain a reliable rendering of the tendon behavior with lumped-parameter models, [11]. On the other hand, with the aim of both simplifying the simulation model and obtaining the same information collected during experiments, the value $N=6$ has been used in this paper. This result has been made possible by a suitable choice of the friction coefficient and of the normal force between the tendon and the sliding surface. In particular, by imposing the equivalence of the tendon tension loss due to the friction along a curvature angle $\varphi=\theta / N$, and by considering the persistence of the sign of the tendon velocity within the considered tendon segment, it possible to define an equivalent friction coefficient as:

$$
\bar{\mu}_{d}(\varphi)=\frac{f_{f}}{\left|\vec{f}_{n}\right|}=\frac{f_{i-1}-f_{i}}{\left|\vec{f}_{i-1}+\vec{f}_{i}\right|}=\frac{1-e^{-\mu_{e} \varphi}}{\alpha\left(1+e^{-\mu_{e} \varphi}\right)}
$$

where $\mu_{e}$ is the friction coefficient identified during the experiments, $f_{i-1}$ and $f_{i}$ are the interaction forces of the $i$-th tendon element with the previous element (or the actuator) and the next element (or the load) respectively, $\alpha$ is the ratio between the module of the vector $\vec{f}_{n}=\vec{f}_{i-1}+\vec{f}_{i}$ and the algebraic sum of the interaction forces $f_{i-1}+f_{i}$. Since the friction coefficient $\bar{\mu}_{d}(\varphi)$ is multiplied by the module of the normal force $\left|\vec{f}_{n}\right|$ in order to obtain the friction force $f_{f}$ and since all the tendon elements are equal in the considered system, a normalized friction coefficient $\mu_{d}$ is used in place of $\bar{\mu}_{d}(\varphi)$ :

$$
\mu_{d}=\frac{1-e^{-\mu_{e} \varphi}}{1+e^{-\mu_{e} \varphi}}
$$

and the normal force $f_{n}=f_{i-1}+f_{i}$ is considered instead of $\left|\vec{f}_{n}\right|$.

\section{THE EXPERIMENTAL SETUP}

The experimental setup is composed by two LinMot$37 \times 160$ linear motors, equipped with customized load cells for measuring the input and output forces applied to the tendon. A low-level control system drives the two linear 

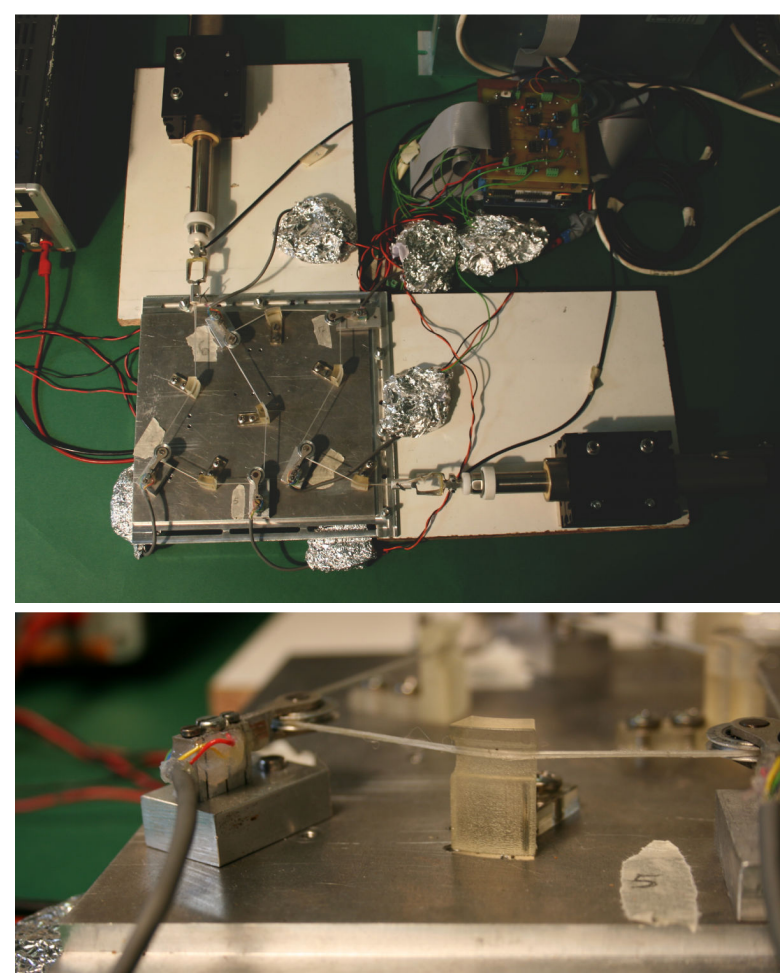

Fig. 5. The experimental setup (top) and detail of the instrumented pulley and of the plastic support (bottom).

motors independently, and gives to these motors a quasiideal and fully-programmable mass/spring/damper behavior compensating also for the friction acting on the motor slider. Moreover, the low-level controller allows the independent control of the resting position (position control mode), or of the interaction forces (force control mode) of the two motors. Due to the symmetry of the actuation system described above, both motors can act as actuator or as load for the tendon transmission system. This fact is exploited in the experiments to evaluate the system behavior alternatively in both directions by exchanging, in some conditions, the actuator with the load and vice versa, as described in Sec. IV.

The tendon path is formed by six identical plastic supports, and each support forms an arc of 30 degrees with a radius of 30 millimeters. The resulting total curvature angle of the tendon subject to friction is $\theta=\pi[\mathrm{rad}]$. In order to measure the tendon tension between two path elements, the tendon is routed through five instrumented pulleys; the working principle of these tendon tension sensors is reported in [13]. The tendon is curved by 30 degrees by each path element, and by 90 degrees by each pulley: it is important to remark that, since the friction arising from pulleys-tendon interaction is negligible with respect to the overall friction, the curvature of the tendon due to the instrumented pulleys does not influence the total curvature angle given by the fixed path. The CAD representation of the tendon path is shown in Fig. 6, while the experimental setup is shown in Fig. 5.

The system is controlled by a PC-104 platform with the RTAI-Linux real time O.S., while a standard PC, running on Linux, is used as development and monitoring platform. The

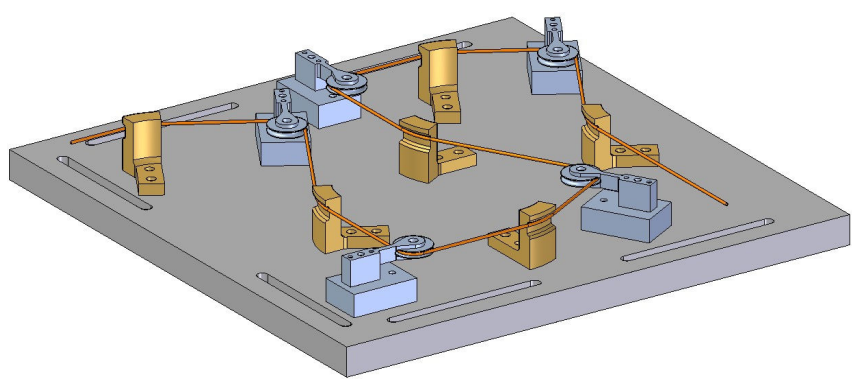

Fig. 6. CAD design of the tendon path equipped with instrumented pulleys and tendon supports.

development system is based on the Matlab/Simulink/RTW environment.

Since the plastic supports acting as friction elements are made in the same material used for the finger in Fig. 1 and since the friction effects depend only on the total tendon curvature angle and they don't scale with the system dimensions, the quite bulky experimental setup that has been implemented reproduces, form the point of view of the friction effects, a scaled version of the tendon path inside the robotic finger, and in particular it allows an accurate study of the tendon transmission behavior avoiding all the problems related to the miniaturization and system integration.

\section{Evaluation of the System Behavior}

The behavior of the tendon-based transmission system has been tested by imposing a sequence of operations to the two linear motors attached to the tendon, and measuring at the same time both the interaction forces between the motors and the tendon at curvature angles 0 and $\pi$ [rad] (by means of the load cells mounted on the motor sliders) and the forces in the tendon tension in the intermediate points at each $\pi / 6$ [rad] of curvature along the path (by means of the instrumented pulleys).

The work flow of the system test is reported in Fig. 7, where the working modality of the two motors are represented as $M_{1}$ and $M_{2}$ respectively, and the initialization of the system parameters during the switching between the two working modalities are indicated by $\mathbb{I}$; the jumps between different states $S_{i}$ are based on the values of the interaction forces of the two motors and the tendon $f_{1}, f_{7}$, and on the time (these conditions are omitted for sake of brevity).

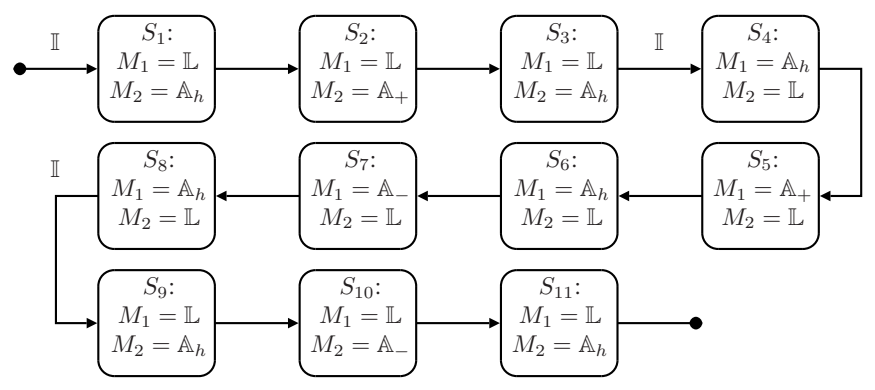

Fig. 7. Work flow of the system test. 


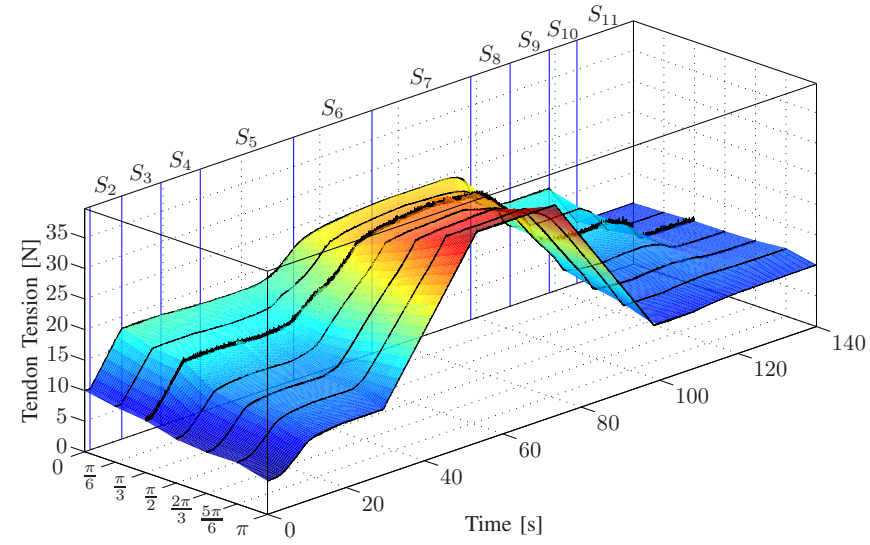

Curvature Angle [rad]

(a) 3D view of the tendon tension distribution with interpolated data

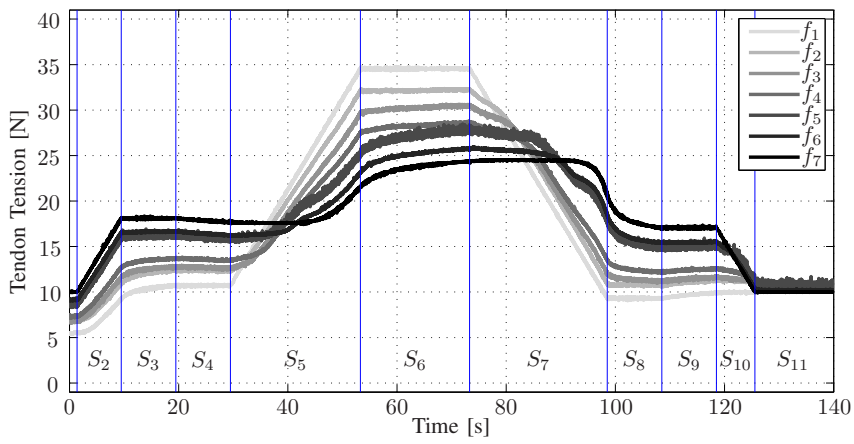

(b) $2 \mathrm{D}$ view of the measured tendon tension distribution.

Fig. 8. Experimental results: tendon tension distribution.

As described in the previous section, each motor can be driven in two different modalities, as actuator $(\mathbb{A})$ that applies a force $f_{c_{j}}$ or as load $(\mathbb{L})$ with a rest position $x_{c_{j}}$, with $j \in\{1,2\}$, see also Fig. 2. It is important to remark that while one motor acts as actuator, the other is acting as load and vice versa.

$\mathbb{A}: \quad f_{c_{j}}$ is commanded, $k_{c_{j}}=0$.

$\mathbb{L}: \quad f_{c_{j}}=0, k_{c_{j}}=k, x_{c_{j}}$ is commanded.

During the test, when a motor is in $\mathbb{A}$ modality, it can be commanded to hold the current interaction force $\left(\mathbb{A}_{h}\right)$, to apply a positive $\operatorname{ramp}\left(\mathbb{A}_{+}\right)$or a negative $\operatorname{ramp}\left(\mathbb{A}_{-}\right)$; moreover, when a motor is switched between the two different modalities (from $\mathbb{A}$ to $\mathbb{L}$ and vice versa) $x_{c_{j}}$ or $f_{c_{j}}$ are properly initialized to avoid undesired jumps in the system:

$$
\mathbb{L} \rightarrow \mathbb{A}: \begin{cases}M_{1}: & f_{c_{1}}=f_{1} \\ M_{2}: & f_{c_{2}}=f_{7} \\ M_{1}: & x_{c_{1}}=x_{m_{1}}-f_{1} / k \\ M_{2}: & x_{c_{2}}=x_{m_{2}}-f_{7} / k\end{cases}
$$

where $x_{m_{j}}$ stays for the actual position of the two motors.

\section{A. Experimental Result}

In this section the data collected by the force sensors during the test described above are presented. The experimental results can be seen in Fig. 8 where the seven measured forces are plotted. In Fig. 8(a) a 3D view of the tendon tension distribution during the test is reported; the colored surface is

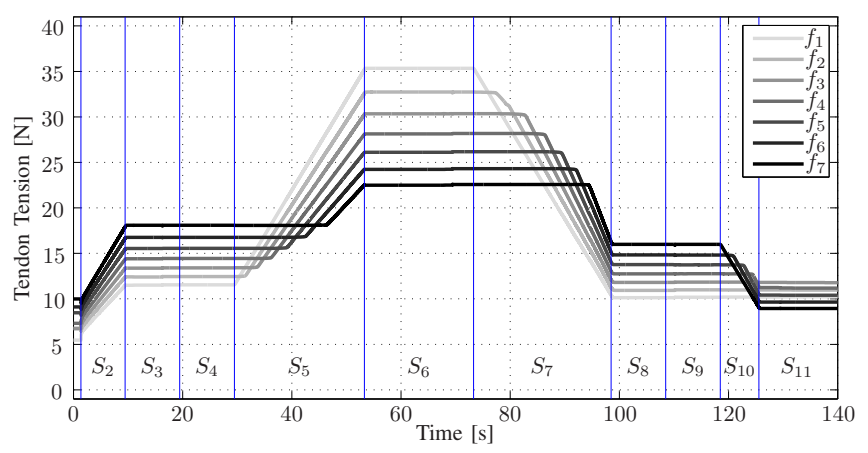

(a) Tension distribution given by Model 1 .

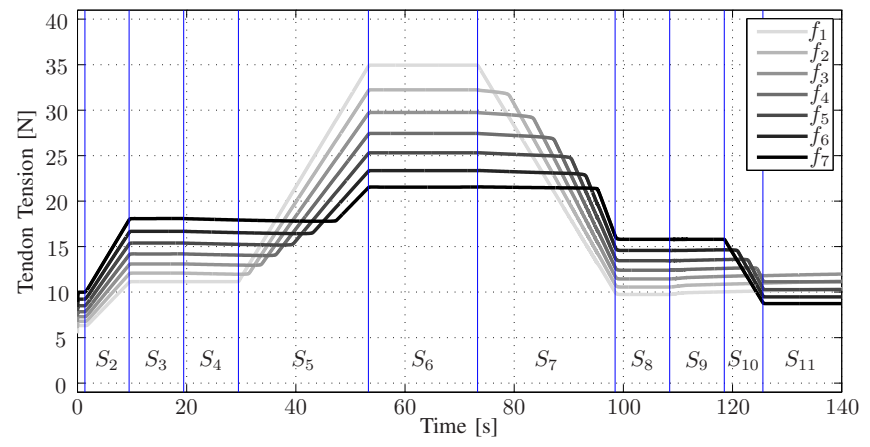

(b) Tension distribution given by Model 2 .

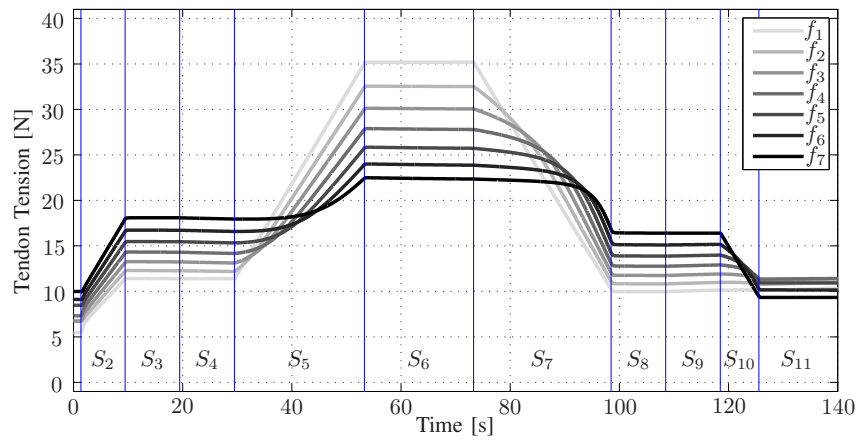

(c) Tension distribution given by Model 3 .

Fig. 9. Simulation results: tendon tension distribution.

obtained trough interpolation of the seven measures of the tendon tension $f_{i}, i \in[1,7]$. In Fig. 8(b) the measured forces are represented in a $2 \mathrm{D}$ view for a simpler comparison with the simulation results.

\section{B. Simulation Results}

Three different models, with increasing level of accuracy in the rendering of the system behavior, have been evaluated with the aim of showing the benefits given by the introduction of additional effects in the simulation models. In the first model only the dry friction phenomena (modeled with the LuGre model with a value of high bristle stiffness $\sigma_{0}$ ) is taken into account, together with a purely elastic tendon behavior. The second model considers (7) as tendon force/deformation relation, thus introducing plasticity. Finally, in the third model the bristle stiffness $\sigma_{0}$ is decreased in order to obtain a smoother hysteresis curve of the friction. 

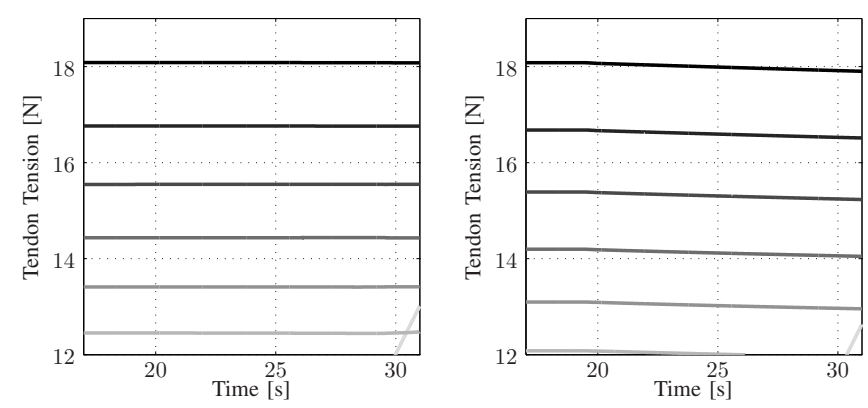

(a) Model 1 (left) Vs Model 2 (right)
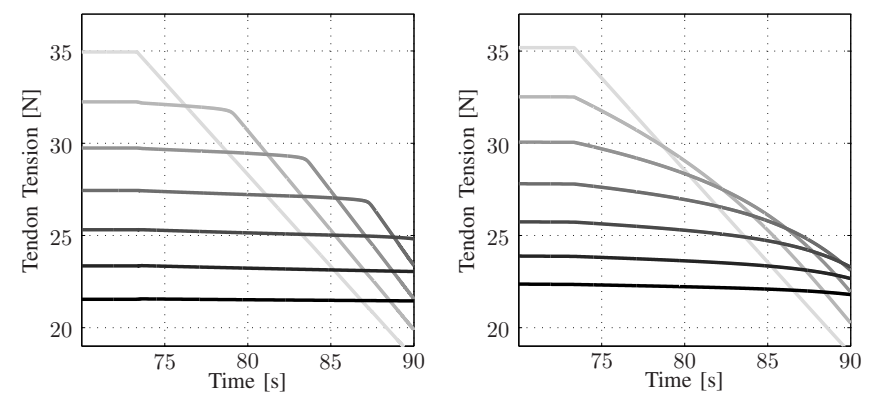

(b) Model 2 (left) Vs Model 3 (right)

Fig. 10. Comparison between simulation results.

The simulation results are reported in Fig. 9: it is possible to note how the resemblance between experimental data and simulation results improves from Fig. 9(a) to Fig. 9(c). In particular, Fig. 9(a) shows the tendon tension distribution obtained with the same simulation model used in [13] to design the tendon friction compensation law, while in Fig. 9(b) the introduction of the plasticity phenomenon described in Sec. II-B results in the tendon relaxation during the states $S_{4}$ and $S_{9}$, as also visible in the experimental data. For sake of clarity, a detailed view of this aspect is reported in Fig. 10(a). Finally, the value of the bristle stiffness has been significantly decreased, obtaining a smoother transient during $S_{5}$ and $S_{7}$. This macroscopic effect is clearly visible in the detailed view reported in Fig. 10(b). This latter model is the most complete among the presented ones, and its results are the closest to the experimental data.

\section{CONCLUSIONS}

In this paper, a detailed investigation of the phenomena that influence the behavior of a tendon-based transmission system has been presented, with particular attention to the effects of friction and plasticity of the tendon. The adoption of a sliding surface or canals to route the tendon from the actuators to the joints of a robotic device, for achieving the maximum simplification and flexibility in the mechanical design, and the use of polymeric fibers as tendon materials, for the ease of the device assembly, are the driving issues of this research activity.

The tendon tension distribution has been measured by means of a suitably designed setup, and the acquired data are compared with the responses of different mathematical models with the aim of finding a good trade-off between the modeling complexity and the fidelity of the obtained results.

The results reported in this paper can be used to improve the design, the development and the control strategies of tendon-driven robotic devices, like robotic hands, endeffectors for robotic surgery, haptic devices, an so on.

Future activities will be devoted to the characterization of different materials for both the tendons and the sliding surfaces, together with the investigation of the effects of lubrication and fatigue on the system behavior.

\section{REFERENCES}

[1] M. Frecker and A. J. Snyder, "Surgical robotics: Multifunctional end effectors for robotic surgery," Operative Techniques in General Surgery, vol. 7, no. 4, pp. $165-169,2005$.

[2] N. Simaan, K. Xu, W. Wei, A. Kapoor, P. Kazanzides, R. Taylor, and P. Flint, "Design and integration of a telerobotic system for minimally invasive surgery of the throat," International Journal of Robotics Research, vol. 28, no. 9, pp. 1134-1153, 2009.

[3] S. Marcheschi, A. Frisoli, C. Avizzano, and M. Bergamasco, "A method for modeling and control complex tendon transmissions in haptic interfaces," in Proc. IEEE Int. Conf. on Robotics and Automation, April 2005.

[4] C. Melchiorri and G. Vassura, "Mechanical and control features of the UB Hand version II," in Proc. IEEE/RSJ Int.Conf.on Intelligent Robots and Systems, IROS'92, 1992.

[5] L. Biagiotti, F. Lotti, C. Melchiorri, G. Palli, P. Tiezzi, and G. Vassura, "Development of UB Hand 3: Early results," in Proc. IEEE Int. Conf. on Robotics and Automation, 2005.

[6] H. Liu, J. Butterfass, S. Knoch, P. Meusel, and G. Hirzinger, "New control strategy for DLR's multisensory articulated hand," IEEE Control Systems Magazine, vol. 19, no. 2, pp. 47-54, 1999.

[7] A. Nahvi, J. M. Hollerbach, Y. Xu, and I. W. Hunter, "Investigation of the transmission system of a tendon driven robot hand," in IEEE International Conference on Intelligent Robots and Systems, 1994, pp. $202-208$.

[8] S. Y. Jung, S. K. Kang, M. Lee, and I. Moon, "Design of robotic hand with tendon-driven three fingers," in Proc. of IEEE Int. Conf. Cont. Aut. and Sys., 2007, pp. 83-86.

[9] G. Berselli, G. Borghesan, M. Brandi, C. Melchiorri, C. Natale, G. Palli, S. Pirozzi, and G. Vassura, "Integrated mechatronic design for a new generation of robotic hands," in Proc. of the IFAC Symposium on Robot Control, Gifu, Japan, 2009.

[10] M. Kaneko, W. Paetsch, and H. Tolle, "Input-dependent stability of joint torque control of tendon-driven robot hands," in Industrial Electronics, IEEE Trans. on, vol. 39, Issue 2, April 1992, pp. 96 104.

[11] G. Palli and C. Melchiorri, "Model and control of tendon-sheath transmission system," in Proc. IEEE Int. Conf. on Robotics and Automation, 2006.

[12] V. Agrawal, W. J. Peine, and B. Yao, "Modeling of a closed loop cableconduit transmission system," in Proc. IEEE Int. Conf. on Robotics and Automation, 2008, pp. 3407-3412.

[13] G. Palli, G. Borghesan, and C. Melchiorri, "Tendon-based transmission systems for robotic devices: Models and control algorithms," in Proc. IEEE Int. Conf. on Robotics and Automation, 2009.

[14] L. L. Howell, Compliant Mechanism. John Wiley \& Sons, 2002.

[15] M. Kaneko, T. Yamashita, and K. Tanie, "Basic considerations on transmission characteristics for tendon drive robots," in Advanced Robotics, 1991. 'Robots in Unstructured Environments', 91 ICAR., Fifth International Conference on, 1991, pp. Page(s):827 - 832 vol.1.

[16] H. Olsson, K. J. Åström, C. C. de Wit, M. Gäfvert, and P. Lischinsky, "Friction models and friction compensation," European Journal of Control, vol. 3, pp. 176-195, 1998.

[17] C. C. de Wit, H. Olsson, K. J. Åström, and P. Lischinsky, "A new model for control of systems with friction," IEEE Trans. on Automatic Control, vol. 40, pp. 419-425, 1995. 